\title{
Membranas de nanocompósitos poliméricos com óxido de zinco para o tratamento de efluentes: Revisão de literatura
}

\author{
Zinc oxide polymeric nanocomposite membranes for wastewater treatment: Literature review \\ Membranas de nanocompuestos poliméricos de óxido de zinc para el tratamiento de aguas \\ residuales: Revisión de la literatura
}

Recebido: 21/06/2021 | Revisado: 28/06/2021 | Aceito: 06/07/2021 | Publicado: 16/07/2021

\author{
José Everton Soares de Souza \\ ORCID: https://orcid.org/0000-0003-0731-534X \\ Universidade Estadual da Paraíba, Brasil \\ E-mail: everton_g3@hotmail.com \\ Bruna Aline Araújo \\ ORCID: https://orcid.org/0000-0002-9429-6994 \\ Universidade Estadual da Paraíba, Brasil \\ E-mail: brunaaaraujo15@gmail.com \\ Kênia Kelly Freitas Sarmento \\ ORCID: https://orcid.org/0000-0001-6815-9577 \\ Universidade Estadual da Paraíba, Brasil \\ E-mail: keniakellys41@gmail.com \\ Larissa Dias Rebouças \\ ORCID: https://orcid.org/0000-0003-3890-6572 \\ Universidade Estadual da Paraíba, Brasil \\ E-mail: larirbcs@gmail.com \\ Keila Machado de Medeiros \\ ORCID: https://orcid.org/0000-0001-9250-1432 \\ Universidade Federal do Recôncavo da Bahia, Brasil \\ E-mail: keilamedeiros@ufrb.edu.br \\ Carlos Antônio Pereira de Lima \\ ORCID: https://orcid.org/0000-0002-1301-6066 \\ Universidade Estadual da Paraíba, Brasil \\ E-mail: caplima@uepb.edu.br
}

\begin{abstract}
Resumo
A necessidade de aplicação de tecnologias mais limpas para o tratamento de efluente e águas são a chave para minimizar os impactos causados ao meio ambiente e recuperação dos recursos hídricos. Dentre essas tecnologias se destacam a tecnologia de membranas pois apresentam vantagens como economia de energia, de fácil operação, substitui os processos convencionais, recupera produtos de alto valor agregado, apresenta flexibilidade no projeto de sistemas e no desenvolvimento de processos híbridos. Este trabalho tem como objetivo realizar uma revisão do processo de separação por membranas, método de obtenção e estudos recentes que utilizam o óxido de zinco (ZnO) como carga inorgânica para obtenção de membranas de nanocompósitos. Membranas híbridas vêm conquistando espaço no meio científico pois apresentam melhorias em propriedade físicas, mecânicas e químicas. A partir dos estudos realizados pelos pesquisadores, foi constatado que o $\mathrm{ZnO}$ vem sendo incorporado como carga para a obtenção de membranas de nanocompósitos poliméricos, pois apresentam melhorias em sua hidrofilicidade, fluxo de água, diminuição da incrustação e as características de resistência ao cloro das membranas obtidas, e desta forma, apresentado potencial para serem aplicadas no tratamento de efluentes.
\end{abstract}

Palavras-chave: Membranas híbridas; Inversão de fases; Nanocompósitos; Tratamento de efluentes.

\begin{abstract}
The need to apply cleaner technologies for effluent and water treatment is the key to minimize the impacts caused to the environment and recovery of water resources. Among these technologies the membrane technology stands out because it has advantages such as energy savings, easy operation, replacing conventional processes, recovering products with high added value, flexibility in the design of systems and in the development of hybrid processes. This paper aims to perform a review of the process of separation by membranes, method of obtaining and recent studies that use zinc oxide $(\mathrm{ZnO})$ as an inorganic load to obtain nanocomposite membranes. Hybrid membranes have been conquering space in the scientific environment because they present improvements in physical, mechanical and chemical properties. From the studies performed by researchers, it was found that $\mathrm{ZnO}$ has been incorporated as a load to obtain polymeric nanocomposite membranes, because it presents improvements in their hydrophilicity, water
\end{abstract}


flow, reduced fouling and chlorine resistance characteristics of the membranes obtained, thus presenting potential to be applied in wastewater treatment.

Keywords: Hybrid membranes; Phase inversion; Nanocomposites; Wastewater treatment.

\section{Resumen}

La necesidad de aplicar tecnologías más limpias para el tratamiento de los efluentes y el agua son la clave para minimizar los impactos causados al medio ambiente y la recuperación de los recursos hídricos. Entre estas tecnologías destaca la tecnología de membranas porque presenta ventajas como el ahorro energético, la facilidad de operación, la sustitución de procesos convencionales, la recuperación de productos de alto valor añadido, la flexibilidad en el diseño de sistemas y el desarrollo de procesos híbridos. Este trabajo pretende realizar una revisión del proceso de separación por membranas, método de obtención y estudios recientes que utilizan el óxido de zinc (ZnO) como carga inorgánica para obtener membranas nanocompuestas. Las membranas híbridas han ido ganando espacio en la comunidad científica porque presentan mejoras en las propiedades físicas, mecánicas y químicas. A partir de los estudios realizados por los investigadores, se ha encontrado que el $\mathrm{ZnO}$ ha sido incorporado como carga para obtener membranas de nanocompuestos poliméricos, ya que presenta mejoras en su hidrofilia, flujo de agua, reducción del ensuciamiento y características de resistencia al cloro de las membranas obtenidas, presentando así potencial para ser aplicadas en el tratamiento de aguas residuales.

Palabras clave: Membranas híbridas; Inversión de fase; Nanocompuestos; Tratamiento de aguas residuales.

\section{Introdução}

Devido ao crescimento populacional e ao desenvolvimento da indústria, há quantidades cada vez maiores de descarte descontrolado de águas residuais; isso não só reduz os recursos de água limpa, mas também causa sérios problemas ambientais e até ameaça à saúde e a segurança dos seres humanos e de outros organismos vivos (Pan et al., 2019). Os efluentes estão contaminados por óleo, sais, matéria orgânica, entre outros contaminantes e o seus descartes só são permitidos depois que estiverem de acordo com a concentração máxima autorizada, e que depende da legislação de cada país. No Brasil, as condições, exigências e padrões de lançamento de efluentes são regulamentados e definidos pela Resolução n 357 e 430 , do Conselho Nacional do Meio Ambiente - CONAMA (Brasil, 2011).

Os processos unitários de separação mais utilizados para tratamento de águas e efluentes são: triagem, decantação, coagulação, floculação, filtração, troca iônica, adsorção, tratamento biológico, desinfecção, oxidação, precipitação química e separações por membranas (Hendricks, 2011). Os tratamentos de efluentes utilizando os processos convencionais tem custo elevado e consome muita energia. Portanto, se faz necessário pesquisas para identificar novas abordagens para purificar a água a um custo menor, utilizando menos energia e, principalmente, minimizando o impacto ambiental.

Neste cenário, destacam-se os processos de separação por membranas (PSM), pois apresentam as seguintes vantagens: economia de energia, tecnologia limpa e de fácil operação, substitui os processos convencionais, recupera produtos de alto valor agregado, apresenta flexibilidade no projeto de sistemas e no desenvolvimento de processos híbridos (Nath, 2017). De forma geral, os processos de separação por membrana são vistos como um conjunto de operações unitárias de separação (Field \& Lipnizki, 2017).

A utilização dos PSM depende de sua área de aplicação como nas indústrias químicas, farmacêuticas, tratamento de águas e efluentes, medicina, entre outras. Os processos que utilizam membranas variam de: microfiltração, ultrafiltração, osmose reversa, diálise, eletrodiálise, permeação de gases e pervaporação (Pabby, Rizvi \& Sastre, 2015).

As membranas comerciais são preparadas a partir de duas classes distintas de materiais: os polímeros que são os orgânicos, e os inorgânicos à base de metal, vidro ou materiais cerâmicos. Normalmente as membranas de natureza orgânica são mais utilizadas por apresentarem menor custo de produção do que as inorgânicas, oferecendo perspectivas de crescimento em termos mercadológicos (Mulder, 1996; Anadão, 2010). As membranas mais utilizadas são produzidas a partir de polímeros sintéticos como as poliamidas, polietersulfona, poliacrilonitrila, polisulfonas, entre outros (Gebreslase, 2018).

A técnica de inversão de fases se apresenta como uma das técnicas mais utilizadas e versáteis para produção de membranas poliméricas assimétricas como as poliamidas (Shaban et al., 2015; Fathollah, 2015). Basicamente, na inversão de 
fases, o polímero é modificado, do estado de solução para o sólido (Kausar, 2017). Este método permite uma grande variação morfológica, a depender do solvente e do não solvente utilizado.

Nanomateriais podem ser incorporados em membranas poliméricas e cerâmicas para melhorar propriedades como rugosidade, permeabilidade e resistência anti-incrustação. Eles podem ser usados incorporados na camada ativa em membranas usadas em tecnologias de tratamento de água e esgoto, em membranas de células de combustível, em membranas de separação de gás ou como componente ativo em tecnologias de remoção de poluentes (Hairom et al., 2014). As membranas híbridas tem capacidade de combinar os componentes inorgânicos e orgânicos durante a formação ou polimerização da membrana, oferecendo vantagens em relação ao tratamento de águas e efluentes (Gohil \& Ray, 2017). A adição de nanopartículas inorgânicas como a argila (de Medeiros et al., 2017; da Silva Barbosa Ferreira et al., 2019), óxido de zinco (ZnO) (Ponnamma et al., 2019), entre outros, em soluções poliméricas para obter membranas híbridas é uma alternativa para a melhoria de propriedades físico-químicas, além de apresentar bom desempenho em diferentes processos de separação e na dessalinização de águas salobras e salinas (Esfahani et al., 2019). O uso de ZnO para obtenção de membranas de nanocompósitos poliméricos é bastante utilizado, pois melhora as propriedades das membranas e tem se mostrado confiável para purificação e descontaminação de água e efluentes domésticos e industriais. Este trabalho tem como objetivo realizar uma revisão de literatura sobre o processo de separação por membranas, método de obtenção e estudos recentes que utilizam o óxido de zinco como inorgânica para obtenção de membranas híbridas.

\section{Metodologia}

A pesquisa foi realizada nas principais bases de dados nacionais e internacionais disponíveis na internet (Sciencedirect, Web of Science, Scielo), contemplando trabalhos acadêmicos e livros-texto especializados e publicados entre 2012 a 2021. Foram utilizadas as bibliografias onde apresentaram maior interesse para o estudo, sem exclusão de trabalhos por ano de publicação. Este estudo trata de uma revisão temática, centrada na utilização de óxido de zinco $(\mathrm{ZnO})$ como carga inorgânica para obtenção de membranas de nanocompósitos poliméricas. Assim, a revisão apresentada é uma síntese de estudos de membranas poliméricas e híbridas com $\mathrm{ZnO}$ para aplicação em microfiltração, ultrafiltração e osmose inversa.

\section{Resultados e Discussão}

\subsection{Processo de Separação por Membranas (PSM)}

Membrana é definida essencialmente como uma barreira, que separa duas fases e restringe o transporte de maneira seletiva (Takht Ravanchi et al., 2009). Os PSM tornaram-se uma alternativa importante para produzir água de boa qualidade, atingindo os padrões de água potável devido à maior taxa de remoção de poluentes orgânicos de baixo peso molecular, minimizando o risco associado à fonte e seus contaminantes, bem como sua modularidade e capacidade para integrar com outros sistemas (Fonseca Couto et al., 2018).

Além da aplicação para tratamento de águas e efluentes, outras aplicações são possíveis como na indústria química, petroquímica, agroalimentar, dessalinização de águas, separação de gases, entre outras (Iulianelli \& Drioli, 2020). A tecnologia de membrana é considerada atraente devido às vantagens como flexibilidade na operação, simplicidade, eficiência energética, compacidade, tamanho reduzido, menor custo operacional e seletividade (Pal et al., 2020).

Os processos de separação por membrana são impulsionados principalmente pela diferença de concentração, diferença de temperatura, diferença de potencial elétrico ou diferença de pressão. A diferença de pressão ou pressão acionada processos são classificados de acordo com o tamanho dos poros dos membrana para quatro processos, conforme apresentado na Tabela 1. Estes processos são microfiltração (MF), ultrafiltração (UF), nanofiltração (NF) e osmose inversa (OI) (Tawalbeh et al., 2018). 
Tabela 1. Processo de separação por membranas.

\begin{tabular}{|c|c|}
\hline Processo & Força motriz \\
\hline Micriofiltração (MF) & $\Delta \mathrm{P}(1-2$ atm $)$ \\
\hline Ultrafiltração (UF) & $\Delta \mathrm{P}(1-7$ atm $)$ \\
\hline Nanofiltração (NF) & $\Delta \mathrm{P}(5-25 \mathrm{~atm})$ \\
\hline Osmose Inversa(OI) & $\Delta \mathrm{P}(15-80 \mathrm{~atm})$ \\
\hline
\end{tabular}

Fonte: Habert; Borges e Nobrega (2006).

As membranas podem ser classificadas em duas categorias: densas ou porosas, de acordo com as características da superfície da membrana que está em contato com a solução de alimentação. Em relação à morfologia elas podem ser isotrópicas (simétricas) ou anisotrópicas (assimétricas), podendo ou não apresentar as mesmas características morfológicas ao longo de sua espessura (Khulbe, Feng \& Matsuura, 2008).

As membranas isotrópicas são conhecidas por possuir estrutura unicamente densa ou porosa com poros de diâmetros regulares ao longo da membrana (Zhu et al., 2018). As membranas anisotrópicas se caracterizam por apresentarem uma região superior muito fina, chamada de camada seletiva, mais fechada com poros muito pequenas ou densa (sem poros), responsável pela seletividade, e por uma estrutura porosa de suporte, que proporciona resistência mecânica e oferece pouca resistência ao transporte. Quando ambas as regiões são constituídas por um único material a membrana é do tipo anisotrópica integral (Torres-Trueba et al., 2008; Park \& Khang, 2016;Wu et al., 2020). Caso materiais diferentes sejam empregados no preparo de cada região, a membrana será do tipo anisotrópica composta. A Figura 1 apresenta um esquema dos diferentes tipos de morfologias das membranas.

Figura 1. Representação dos diferentes tipos de morfologia das membranas.

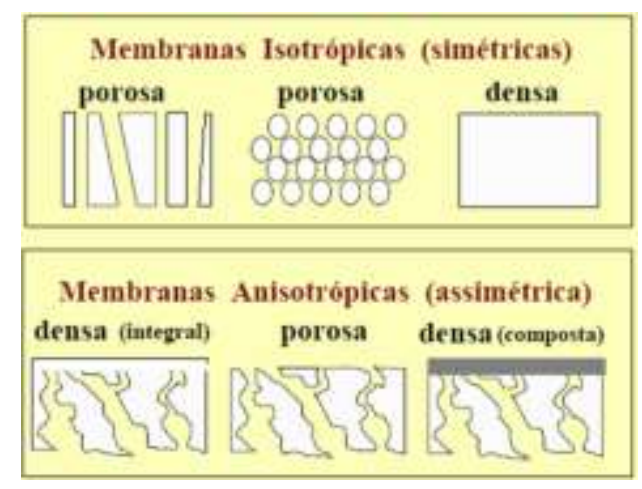

Fonte: Habert; Borges e Nobrega (2006).

As membranas isotrópicas densas consistem em um filme não poroso de diâmetro de poros muito menor que $0,1 \mathrm{~nm} \mathrm{e}$ geralmente tem menos de $20 \mu \mathrm{m}$ de espessura, através do qual as misturas de moléculas são transportadas por sorção e difusão. Como o fluxo obtido dessas membranas é baixo, sua utilização é restrita a separação de gases e pervaporação. As membranas isotrópicas microporosas consistem em poros uniformes em toda a membrana e contém um tamanho de poros variando entre 0,01 a $10 \mu \mathrm{m}$. Essas membranas são usadas como filtros, pois partículas maiores que o tamanho dos poros é retida na superfície da membrana, permitindo a passagem de apenas alguns componentes. Essas membranas são caracterizadas por porosidade, diâmetro dos poros e tortuosidade (Moulik \& Sridhar, 2019).

As membranas anisotrópicas possuem uma camada ativa densa (espessura $<1 \mu \mathrm{m}$ ) e uma camada de suporte poroso (espessura 100-150 $\mu \mathrm{m}$ ) que consiste do mesmo material polimérico. Por outro lado, membranas nas quais essas camadas são feitas de diferentes materiais poliméricos são chamados de membranas compostas. A camada de pele densa da membrana 
anisotrópica composta é a camada ativa para a separação e a camada de esponja porosa tem a função de suporte para a camada de pele densa. Nas membranas compostas, uma vez que a camada ativa densa e a camada de suporte poroso consistem em materiais diferentes, a flexibilidade no design mais adequado dessas camadas é significativamente alta em comparação com as membranas anisotrópicas (Uragami, 2017).

\subsection{Materiais para Fabricação de Membranas}

Os materiais utilizados para obtenção de membranas sintéticas são diferenciados em duas classes: os inorgânicos e orgânicos. De forma geral, as membranas inorgânicas consistem em membranas cerâmicas, metálicas e membranas à base de carbono. Óxidos como alumina, sílica, titânia ou as misturas desses componentes são algumas das membranas cerâmicas comumente comercializadas. Do ponto de vista econômico e de manutenção, as membranas inorgânicas são ideais para limpeza química no local, onde altas temperaturas e limpeza química subsequente podem facilmente gerar fluxo de recuperação sustentável. Além disso, são menos suscetíveis à deterioração por bactérias (Goh \& Ismail, 2018).

Os principais materiais para a formação das membranas orgânicas, em sua maioria, são polímeros, como acetato de celulose (AC), poliamida (PA), polieterimida (PEI), poliimida (PI), polisulfona (PS) e poliétersulfona (PES). As membranas de AC e PI geralmente possuem estrutura isotrópica, já as membranas fabricadas de PA, PES e híbridas apresentam estrutura anisotrópica (Agboola et al., 2020). Os polímeros são os materiais mais atraentes na indústria de membranas devido às suas diversidades e métodos simples de fabricação (Yong \& Zhang, 2021).

\subsection{Obtenção de Membranas Microporosas Poliméricas}

A principal técnica de preparo de membranas microporosas poliméricas é a inversão de fases que pode ser efetuada por diferentes métodos, incluindo, precipitação por evaporação de solvente, precipitação térmica e precipitação por imersão. Esta técnica é bastante utilizada para preparar membranas porosas e não porosas. A maioria das membranas comerciais poliméricas é produzida pela técnica de inversão de fases, devido a sua facilidade de obtenção e baixo custo (Naz et al., 2020).

No método de precipitação por evaporação, o polímero é dissolvido em uma mistura de um solvente volátil e um não solvente menos volátil que induz a queda da solubilidade do polímero, acarretando na separação de fase. Na precipitação em presença de vapores a separação de fase é motivada pela exposição da solução polimérica numa atmosfera que contém vapores do próprio solvente e de um não solvente (Altun et al., 2017). A precipitação térmica caracteriza-se por induzir instabilidade a uma solução polimérica através da aplicação de elevada temperatura seguida de resfriamento, onde ocorre a diminuição da solubilidade do polímero no solvente. O solvente é removido ao final por extração, evaporação ou secagem (Matsuyama et al., 2002). Já a precipitação por imersão, caracteriza-se por precipitar uma solução polimérica, na forma de filme fino ou extrusada na forma de fibra oca, em um banho de não solvente. Neste processo, a solução polimérica homogênea é separada em duas fases: uma fase sólida rica em polímero, a qual forma a matriz da membrana e uma fase líquida pobre em polímero a qual forma a estrutura porosa. Após a imersão da solução polimérica no banho de coagulação contendo o não solvente, este irá difundir para a solução, enquanto o solvente se difundirá no banho, conforme ilustrado na Figura 2. A força motriz para este processo é a diferença de potencial químico existente entre o filme e o banho. A transferência de massa entre o solvente e o não solvente é um fator determinante na morfologia e nos fenômenos envolvidos na síntese de membranas (Bellincanta et al, 2011). 
Figura 2. Obtenção de uma membrana plana pelo método de precipitação por imersão.

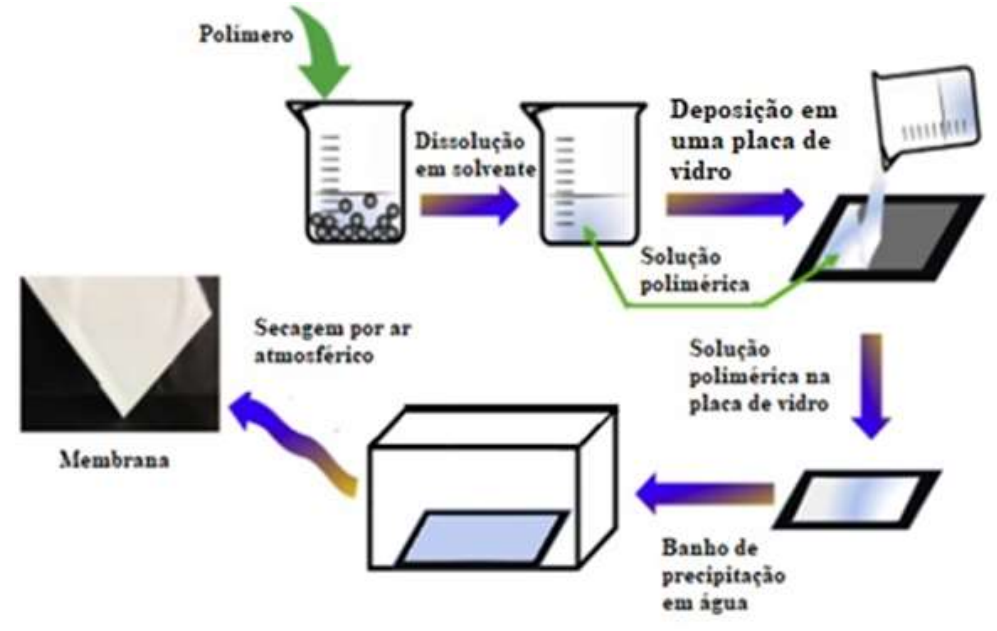

Fonte: Adaptada de Chinyerenwa et al. (2018).

O tamanho e a distribuição do poro da membrana são determinados pela termodinâmica e cinética da inversão de fase intimamente relacionada com as propriedades da composição da solução de polímero e não solvente, e as condições de operação. O profundo conhecimento do processo de inversão de fase é valioso para ajustar e controlar com eficácia a estrutura da membrana e as funcionalidades de anti-incrustação, seletividade e fluxo (He et al., 2011).

\section{4 Óxido de Zinco (ZnO)}

O óxido de zinco $(\mathrm{ZnO})$ é um semicondutor, encontrado naturalmente no mineral chamado zincita e está na classe dos óxidos condutores transparente, é um pó fino, amorfo, de coloração branca e com densidade igual a 5,6 g.cm ${ }^{-3}$ (Mayrinck et al, 2014). Além disso, possui propriedades interessantes como uma alta energia de ligação, excelente estabilidade química, amplo gap de banda, não tóxico, hidrofilicidade comutável, facilmente dissolvido em ácidos, boa robustez física, entre outros (Mishra \& Adelung, 2018; Ghanbari Shohany \& Khorsand Zak, 2020).

$\mathrm{O} \mathrm{ZnO}$ pode apresentar três estruturas, a sal rocha, a blenda de zinco e a wurtzita, sendo que essa última é a única termodinamicamente estável em condições ambientes e, portanto, a sua síntese não necessita de condições especiais. A estrutura sal rocha é obtida a elevadas pressões, e o tipo blenda de zinco somente se estabiliza por crescimento em substratos cúbicos. A wurtzita de $\mathrm{ZnO}$ consiste em uma estrutura hexagonal, na qual, em sua célula unitária (Figura 3), cada átomo de zinco está no centro de um tetraedro coordenado a quatro outros átomos de oxigênio (Ozgur et al., 2005).

Figura 3. Célula unitária representando a estrutura hexagonal da wurtzita de $\mathrm{ZnO}$.

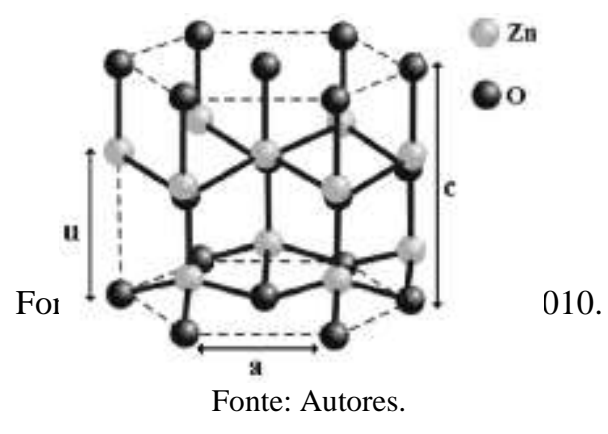


A maior parte usada comercialmente de $\mathrm{ZnO}$ é produzida através de métodos sintéticos, dedicados à formação de nanopartículas de $\mathrm{ZnO}$, como: síntese hidrotérmica, precipitação alcalina, decomposição térmica e método sol-gel (Zang \& Tang, 2015;Mirzaei \& Darroudi, 2017). As aplicações do ZnO são bastante variadas devido às suas características, tais como: emissores de luz ultravioleta, dispositivos piezoelétricos, sensores químicos e de gás, transistores, células de energia solar, catalisadores, medicamentos, fotocatalisadores para inativar bactérias e para a degradação de poluentes ambientais (Kahouli et al., 2015; Obreja et al., 2019). Devido às suas características, o ZnO vem sendo incorporado como aditivo para obtenção de membranas híbridas, apresentando melhorias em suas hidrofilicidade, fluxo de água, diminuição da incrustação e as características de resistência ao cloro das membranas (Rajakumaran et al., 2019b).

\subsection{Membranas de Nanocompósitos Poliméricos com Óxido de Zinco}

A partir do estudo na literatura, foi possível identificar vários tipos de processos de separação por membranas que utilizaram $\mathrm{ZnO}$ como nanocompósitos com aplicação em microfiltração, ultrafiltração e osmose inversa.

El-Arnaouty et al., (2018) desenvolveram membranas de poliamida para osmose inversa (OI), com o enxerto de nanopartículas de $\mathrm{N}$-isopropil acrilamida (NIPAM) e ZnO. Foi investigado o efeito da concentração de monômeros, tempo de radiação e concentração de $\mathrm{ZnO}$ sobre a porcentagem do enxerto. As propriedades das membranas foram caracterizadas por ângulo de contato (AC), FTIR, DRX e MEV. O desempenho das membranas de PA pura e modificada em termos de fluxo de água e rejeição de sal, a bioincrustação e o cloro nas propriedades de resistência foram avaliados. Verificou-se que a membrana modificada com ZnO-NIPAM é muito melhor quando comparada com a membrana de PA pura.

Isawi, (2018) investigou a nanoestrutura superficial da membrana composta de filme fino (TFC) de poliamida PA que foi aprimorada para dessalinização da água por OI, usando nanocompósitos $(\mathrm{NCs})$ sintetizados $\left(\mathrm{ZnO} / \mathrm{TiO}_{2}\right)$ incorporados à solução de enxerto de ácido metacrílico (MAA) hidrofílico. Os NCs sintetizadas $\left(\mathrm{ZnO} / \mathrm{TiO}_{2}\right)$ e as propriedades de superfície das membranas foram caracterizadas por FTIR, DRX, MEV e AC. Além disso, o desempenho da membrana também foi avaliado. Os resultados preliminares indicam que as membranas de $\mathrm{NCs}$ com $\mathrm{ZnO} / \mathrm{TiO}_{2}$ apresentaram melhores resultados em termos de fluxo de água, rejeição de sal, propriedades mecânicas, resistências de incrustação, estabilidade em soluções alcalinas ácido-alcalinas e propriedades de anti-incrustação biológica do PA (TFC). A membrana dos $\mathrm{NCs}\left(\mathrm{ZnO} / \mathrm{TiO}_{2} / \mathrm{PMAA}\right)$ -g-PA (TFC) apresentou rejeição de sal de $96,8 \%$ e fluxo de água de $33,3 \mathrm{~L} \cdot \mathrm{m}^{2} \cdot \mathrm{h}^{-1}$ usando pressão de 15 bar e uma solução salina de cloreto de sódio $(\mathrm{NaCl})$ de $2000 \mathrm{mg} \cdot \mathrm{L}^{-1}$. Além disso, a membrana modificada aumentou consideravelmente a resistência à incrustação da albumina de soro bovino (BSA) como uma incrustação potencial e inibiu a ligação microbiana e o crescimento de biofilme nas superfícies da membrana usando Escherichia coli (E. coli) como modelo de incrustação.

Dinari e Haghighi, (2018) analisaram nanocompósitos (NCs) de poliamida aromática (PA) introduzindo ZnO modificadas com s-triazina (TSC), na superfície para remoção eficiente de cromo hexavalente tóxico (VI) da solução aquosa. Os NCs sintetizados PA/ZnO-TSC (Figura 4) foram caracterizados pelos métodos espectroscopia de infravermelho com transformada de Fourier (FTIR), difração de raios-X (DRX), MEV, microscopia eletrônica de transmissão (MET) e termogravimetria (TG). Imagens de MET mostraram que as nanopartículas de $\mathrm{ZnO}$ estavam dispersas homogeneamente na matriz polimérica. As remoções máximas de $\mathrm{Cr}$ (VI) em pH 4,0 foram de 72\%, 81\%, 89\% e 91\% para PA puro, NC5\%, $\mathrm{NC} 10 \%$ e NC15\%, respectivamente. A cinética da adsorção foi investigada e o modelo de pseudo-segunda ordem é um modelo apropriado para interpretação do mecanismo de adsorção de íons Cr (VI). 
Figura 4. Interações entre PA aromático e nanocompósitos de TSC-ZnO e possível mecanismo de adsorção de íons metálicos por nanocompósitos de PA/TSC-ZnO.

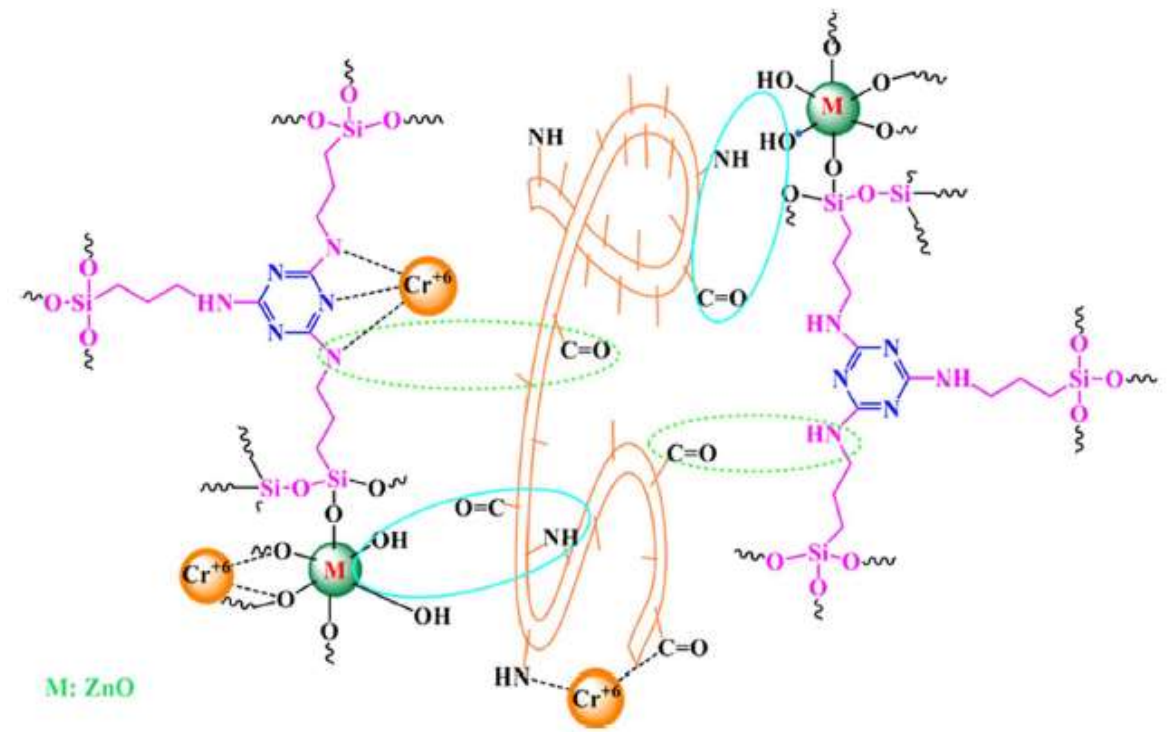

Fonte: Dinari et al. (2018).

Rajakumaran et al., (2019) avaliaram membrana para OI de nanocompósitos de película fina (TFN) com óxido de grafeno (GO) e amino-funcionalizado com $\mathrm{ZnO}$ com diferentes morfologias, isto é, esféricas (ZnO-S), flor (ZnO-F) e haste (ZnO-R) nanoestruturada em forma. As propriedades da superfície do TFN foram investigadas usando MEV, MET, DRX, FTIR, microscopia de força atômica (AFM), espectroscopia de fotoelétrons excitados por raios X (XPS) e AC. O desempenho da membrana foi avaliado usando uma configuração de filtração de fluxo cruzado a $25^{\circ} \mathrm{C}$ e pressão de 20 bar para solução de $\mathrm{NaCl} 2000$ mg.L $\mathrm{L}^{-1}$. Os resultados experimentais indicaram que 0,02\% em peso de GO-ZnO nas membranas, independentemente da sua forma, exibiram hidrofilicidade, fluxo e permeabilidade aprimorados. A membrana modificada com uma concentração ótima de $\mathrm{GO}-\mathrm{ZnO}$ de $0,02 \%$ em peso apresentou maior fluxo de água do soluto $\left(31,42 \mathrm{~L}^{-1} \mathrm{~h}^{-1} \cdot \mathrm{m}^{2}\right)$ em comparação com a membrana sem modificação $\left(14,28 \mathrm{~L} \cdot \mathrm{h}^{-1} \cdot \mathrm{m}^{2}\right)$ com uma boa rejeição de sal. Além disso, as membranas de nanocompósitos foram resistentes ao cloro e apresentaram melhor desempenho anti-incrustante em comparação com a membrana sem modificação.

Amini et al., (2020) estudaram membranas de nanocompósitos de PA/ZnO pela técnica de inversão de fases. O método de polimerização interfacial foi utilizado para a preparação dos nanocompósitos. O efeito da introdução das nanopartículas de $\mathrm{ZnO}$ foi estudada por FTIR, MFA e MEV. Além disso, o fluxo de água e a hidrofilicidade das membranas foram investigadas usando uma solução de cloreto de sódio e AC, respectivamente. A partir dos resultados foi constatado que ocorreu um aumento notável na hidrofilidade, porosidade e no fluxo de água das membranas, após a adição de ZnO. Por outro lado, quanto maior o percentual de $\mathrm{ZnO}$ menor foi o ângulo de contato obtido. Todas as membranas de nanocompósitos com destaque para a de $0,5 \%$ de $\mathrm{ZnO}$ exibiram um alto desempenho se comparado com a membrana de PA, evidenciando assim, que estas membranas apresentaram potencial para a dessalinização de águas.

Rajakumaran et al., (2020) estudaram o efeito de nanoestruturas de formato controlado na eficiência de dessalinização de membranas de nanocompósito de filme fino (NFT). Nanoestruturas de ZnO como nanoflores (F-ZnO), nanobastões (R$\mathrm{ZnO})$ e nanopartículas esféricas $(\mathrm{S}-\mathrm{ZnO})$ foram sintetizadas usando processos hidrotérmicos e sol-gel. As características químicas e estruturais das nanopartículas e das membranas NFT foram analisadas usando DRX, FTIR, MEV, medição do ângulo de contato e MFA. O desempenho de dessalinização das membranas NFT foi investigado após a incorporação de diferentes concentrações de nanopartículas de $\mathrm{ZnO}$ ( $\mathrm{S}-\mathrm{ZnO}$, F-ZnO e R-ZnO) variando de 0 a $0,03 \%$ em peso. 
Independentemente da morfologia de $\mathrm{ZnO}$, a membrana NFT mostrou hidrofilicidade melhorada com um aumento na carga de ZnO. A membrana S- ZnO/0,02 TFN exibiu o maior fluxo de permeado $\left(23,85 \mathrm{~L} \cdot \mathrm{m}^{-2} \cdot \mathrm{h}^{-1}\right)$ e rejeição de $\mathrm{NaCl}$ relativamente melhor (97\%), o que pode ser atribuído à maior área de superfície e menor tamanho de S-ZnO em comparação com FNanoestruturas de $\mathrm{ZnO}$ e R-ZnO. Além disso, o estudo anti-incrustante (com cepa DH5-alfa, Escherichia coli) para a membrana S-ZnO/0,02 NFT revelou uma redução nas células viáveis para 70\% após 180 min de contato em comparação com a membrana composta de película fina (CFT). A membrana S-ZnO/0,02 NFT exibiu estabilidade operacional aprimorada, o que confirma a incorporação estável de nanopartículas de S-ZnO na camada seletiva de poliamida.

Kazemi et al., (2021), nanopartículas GO (óxido de grafeno) e GO-ZnO foram incorporadas às membranas de PVC para aumentar as propriedades anti-incrustantes no tratamento de águas residuais oleosas. Os resultados da análise de FTIR revelaram que as nanopartículas de $\mathrm{ZnO}$ foram ancoradas com sucesso em nanofolhas GO. Além disso, os resultados de XRD indicaram que a presença de $\mathrm{ZnO}$ aumentou a distância entre as nanofolhas GO. As membranas fabricadas foram caracterizadas por ângulo de contato, ensaio de tração, fluxo de água pura, microscópio eletrônico de varredura por emissão de campo (FESEM) e microscopia de força atômica (AFM). Os resultados do FESEM ilustraram que a modificação do GO com $\mathrm{ZnO}$ reduziu sua aglomeração na matriz da membrana. A redução do ângulo de contato de $80^{\circ}$ para a membrana de PVC puro para $67^{\circ}$ para a membrana contendo $0,1 \%$ de GO-ZnO mostrou que a hidrofilicidade das membranas foi aumentada pelo aumento do conteúdo de nanopartículas. A mesma tendência foi observada para fluxo de água de membranas de nanocompósitos de PVC/GO e PVC/GO-ZnO, indicando que membranas com 0,05\% em peso de GO e GO-ZnO exibiram o maior fluxo de água pura. A presença de nano aditivos também aumentou a resistência mecânica das membranas devido à propriedade sinérgica dos nano aditivos. As membranas foram avaliadas em um sistema de fluxo cruzado em escala de laboratório com água oleosa como alimentação. Os resultados mostraram que as membranas de PVC/GO-ZnO apresentaram menor redução de fluxo e maior eficiência de remoção de turbidez do que as membranas de PVC puro e PVC/GO.

Amini et al., (2020), estudaram a membrana de nanocompósito de poliamida (PA) com óxido de zinco (ZnO), que foi fabricada incorporando várias quantidades de nanopartículas de $\mathrm{ZnO}$ (variando de zero a 1,0\% em peso) na matriz da membrana. O método de polimerização interfacial foi usado para preparar membranas de nanocompósitos de filme fino (TFN) para aplicação de osmose direta (FO). O efeito das nanopartículas de $\mathrm{ZnO}$ nas estruturas e composições químicas das membranas sintetizadas foi estudado usando espectroscopia FT-IR, microscópio de força atômica (AFM) e microscopia eletrônica de varredura (MEV). Além disso, o desempenho das membranas fabricadas (fluxo de água) e a hidrofilicidade foram investigados usando solução de pó de $\mathrm{NaCl}$ e medidas de ângulo de contato, respectivamente. Resultados experimentais mostraram que o fluxo de água das membranas preparadas aumentou notavelmente após a adição de nanopartículas de $\mathrm{ZnO}$. Todas as membranas TFN preparadas exibiram um desempenho de dessalinização aprimorado em sistemas FO em comparação com a membrana de poliamida composta de película fina original (TFC).

Nasrollahi et al., (2018) desenvolveram membranas de ultrafiltração de polietersulfona (PES) misturadas com diferentes conteúdos dos nanocompósitos $\mathrm{CuO} / \mathrm{ZnO}(\mathrm{CZN})$ foram preparadas pelo método de inversão de fase não induzida por solvente. A alta hidrofilia da nanopartícula de $\mathrm{ZnO}$ a tornou uma excelente escolha para ser incorporada com nanopartículas de $\mathrm{CuO}$, a fim de melhorar as propriedades da membrana como um aditivo de nanocondicionamento. Microscopia eletrônica de varredura (MEV), microscopia de força atômica (MFA), medição do tamanho médio dos poros, ângulo de contato com a água e raios-X dispersivos de energia (EDX) foram usados para caracterização da membrana. Imagens de MEV em corte transversal mostraram que pela adição de CZN até $0,2 \%$ em peso, a morfologia da membrana teve pouca tendência para alterar de uma estrutura semelhante a um dedo para um macro-vazio. No entanto, ao aumentar ainda mais o CZN, a porosidade foi reduzida. A distribuição homogênea e a ausência de aglomeração de CZN, que podiam ser observadas nas imagens SEM de superfície, eram o resultado da dependência adequada entre o CZN e a matriz polimérica. A análise de 
EDX apresentou a dispersão homogênea das nanopartículas na matriz PES. Imagens de AFM também mostraram que a rugosidade da superfície não foi alterada significativamente pela concentração de CZN. No entanto, os parâmetros de rugosidade calculados mostraram que a adição de CZN até o valor ideal $(0,2 \%$ em peso) pode ser eficaz na redução da rugosidade da superfície da membrana. A membrana de matriz mista até o valor ótimo de CZN (0,2\% em peso) apresentou as propriedades melhoradas de permeabilidade à água e resistência à incrustação. Quando o conteúdo de CZN era de 0,2\% em peso, o crescimento do fluxo de água pura da membrana combinada era de cerca de $32 \%$. Além disso, a membrana combinada citada exibiu uma razão de recuperação de fluxo mais alta (FRR (\%)) no agente de incrustação de albumina de soro bovino (BSA). A rejeição de BSA foi acima de 95\% para todas as membranas de nanocompósitos.

Leo et al., (2012) estudaram as membranas de polissulfona (PSf) são hidrofóbicas, portanto, são suscetíveis à incrustação pela maioria dos componentes orgânicos, incluindo ácidos graxos. Neste trabalho, nanopartículas de ZnO foram misturadas em membranas de PSf para melhorar suas propriedades na redução de incrustação por ácido oleico. A solução de fundição foi preparada dispersando 1-4\% em peso de nanopartículas de $\mathrm{ZnO}(21-23 \mathrm{~nm}), 1 \%$ em peso de Álcool polivinílico (PVA) e PSf em 1-metil-2-pirrolidinona a $75{ }^{\circ} \mathrm{C}$, com uma velocidade de agitação de $400 \mathrm{rpm}$. A proporção em peso de polímero para solvente é mantida a 1: 4,84. As membranas compostas foram então preparadas usando o método de inversão de fase. O tamanho médio dos poros das membranas compostas é ligeiramente maior do que o tamanho médio dos poros da membrana PSf pura. No entanto, o ângulo de contato com a água reduz muito de $85^{\circ}$ para $63^{\circ}$ quando $2 \%$ em peso de $\mathrm{ZnO}$ é adicionado às membranas Pf-2. A estabilidade térmica das membranas PSf melhorou significativamente com a adição de $\mathrm{ZnO}$. Embora os picos de absorção de $\mathrm{ZnO}$ estejam ausentes nos padrões de FT-IR, a análise de EDX mostra que as nanopartículas de $\mathrm{ZnO}$ estão bem distribuídas na camada densa da membrana Pf-2. Portanto, as membranas Pf-2 mostram a maior permeabilidade e a menor incrustação por ácido oleico.

Zhang et al., (2014) estudaram membranas de polivinilidenefluoreto ( $\mathrm{PVDF} / \mathrm{ZnO}$ ) hibridizadas com $\mathrm{ZnO}$ foram preparadas com sucesso por dois métodos diferentes. Um método foi imergir os filmes de PVDF pré-tratados nas suspensões de $\mathrm{ZnO}$ (método A), e o outro foi misturar nanopartículas de $\mathrm{ZnO}$ com solução de PVDF e, em seguida, moldar os filmes (método B). A estrutura dessas membranas híbridas PVDF/ZnO foi caracterizada por MEV, DRX e FTIR, e o desempenho das membranas híbridas foi determinado através das medidas de ângulo de contato, fluxo de água pura e adsorção estática para Albumina sérica bovina. Os resultados mostraram que as nanopartículas de $\mathrm{ZnO}$ foram incorporadas nos poros e na superfície do PVDF, e uma estrutura híbrida mais uniforme foi obtida pelo método B. Os espectros FTIR revelaram que a fraca interação física desempenhou papel na construção de membranas híbridas. Em comparação com os filmes de PVDF originais, a hidrofilia, a permeabilidade e o desempenho anti-incrustação das membranas híbridas foram melhorados. E mais notavelmente, as membranas híbridas também mostraram melhores propriedades de adsorção e de dessorção para íons de cobre.

\section{Considerações Finais}

Os processos de separação por membranas são bastante utilizados no tratamento de efluentes, contudo, novos estudos são apresentados com a utilização de membranas híbridas para otimização das propriedades das membranas. Diante do exposto, a utilização do óxido de zinco como carga inorgânica para ser incorporado em polímeros e, posterior obtenção das membranas de nanocompósitos poliméricos, tem recebido considerável atenção devido às suas características físico-químicas favoráveis ao processo de separação. A partir dos estudos elencados, nota-se que as membranas híbridas com ZnO aumentaram as propriedades de fluxo, hidrofilicidade e permeabilidade, quando se comparado com membranas puras, encontrando rendimentos que variaram de $91 \%$ para remoção de cromo hexavalente tóxico $\mathrm{Cr}$ (VI), chegando a uma rejeição de sal de até 96,8\% de uma solução contendo cloreto de sódio. As propriedades de anti-incrustação, de adsorção e de rejeição de sais também foram otimizadas quando adicionado o $\mathrm{ZnO}$, apresentando dessa forma um grande potencial para aplicação em 
processos de separação por microfiltração, ultrafiltração, nanofiltração e osmose inversa e, consequentemente, serem utilizadas no tratamento de águas e efluentes, além de apresentar uma grande oportunidade para estudos futuros, relacionando o $\mathrm{ZnO}$ com outras cargas inorgânicas com intuito de melhorar as propriedade físico-químicas de membranas poliméricas.

\section{Agradecimentos}

O presente trabalho foi realizado com apoio da Universidade Estadual da Paraíba - UEPB. Além disso, os autores agradecem ao Grupo de Pesquisa em Tratamentos Avançados de Águas - GRUTAA/UEPB, ao Conselho Nacional de Desenvolvimento Científico e Tecnológico - CNPq e à Coordenação de Aperfeiçoamento de Pessoal de Nível Superior CAPES pelas bolsas concedidas.

\section{Referências}

Agboola, O., Sunday Isaac Fayomi, O., Sadiku, R., Popoola, P., Adeniyi Alaba, P., \& Adegbola, A. T. (2020). Polymers blends for the improvement of nanofiltration membranes in wastewater treatment: A short review. Materials Today: Proceedings, 43, 3365-3368. https://doi.org/10.1016/j.matpr.2020.05.387

Altun, V., Remigy, J. C., \& Vankelecom, I. F. J. (2017). UV-cured polysulfone-based membranes: Effect of co-solvent addition and evaporation process on membrane morphology and SRNF performance. Journal of Membrane Science, 524, 729-737. https://doi.org/10.1016/j.memsci.2016.11.060

Amini, M., Seifi, M., Akbari, A., \& Hosseinifard, M. (2020). Polyamide-zinc oxide-based thin film nanocomposite membranes: Towards improved performance for forward osmosis. Polyhedron, 179, 114362. https://doi.org/10.1016/j.poly.2020.114362

Anadão, P. (2010). Ciência e Tecnologia de Membranas. Artliber Editora Ltda.

Bellincanta, T., Poletto, P., Thürmer, M. B., Duarte, J., Toscan, A. Zeni, M. (2011). Preparação e caracterização de membranas poliméricas a partir da blenda polisulfona/ poliuretano. Polímeros, v. 21, p. 229-232.https://doi.org/10.1590/S0104-14282011005000045

Brasil, Lei. Ministério do Meio Ambiente. Conselho Nacional de Meio Ambiente - Conama. (2011). Resolução nº 430, de 13 de maio de 2011. Dispõe sobre as condições e padrões de lançamento de efluentes e altera a Resolução n ${ }^{\circ} 357$. http://www.suape.pe.gov.br/images/publicacoes/CONAMA_n.430.2011.pdf

Chinyerenwa, A. C., Wang, H., Zhang, Q., Zhuang, Y., Munna, K. H., Ying, C., Yang, H., \& Xu, W. (2018). Structure and thermal properties of porous polylactic acid membranes prepared via phase inversion induced by hot water droplets. Polymer, 141, 62-69. https://doi.org/10.1016/j.polymer.2018.03.011

da Silva Barbosa Ferreira, R., Oliveira, S. S. L., Salviano, A. F., Araújo, E. M., Leite, A. M. D., \& de Lucena Lira, H. (2019). Polyethersulfone hollow fiber membranes developed for oily emulsion treatment. Materials Research, 22, 1-8. https://doi.org/10.1590/1980-5373-MR-2018-0854

de Medeiros, K. M., Araújo, E. M., Lira, H. de L., Lima, D. de F., \& Lima, C. A. P. de. (2017). Membranas microporosas híbridas assimétricas: Influência da argila na morfologia das membranas. Revista Materia, 22(2). https://doi.org/10.1590/S1517-707620170002.0144

Dinari, M., \& Haghighi, A. (2018). Ultrasound-assisted synthesis of nanocomposites based on aromatic polyamide and modified ZnO nanoparticle for removal of toxic Cr(VI) from water. Ultrasonics Sonochemistry, 41(July 2017), 75-84. https://doi.org/10.1016/j.ultsonch.2017.09.023

El-Arnaouty, M. B., Abdel Ghaffar, A. M., Eid, M., Aboulfotouh, M. E., Taher, N. H., \& Soliman, E.-S. (2018). Nano-modification of polyamide thin film composite reverse osmosis membranes by radiation grafting. Journal of Radiation Research and Applied Sciences, 11(3), 204-216. https://doi.org/10.1016/j.jrras.2018.01.005

Esfahani, M. R., Aktij, S. A., Dabaghian, Z., Firouzjaei, M. D., Rahimpour, A., Eke, J., Escobar, I. C., Abolhassani, M., Greenlee, L. F., Esfahani, A. R., Sadmani, A., \& Koutahzadeh, N. (2019). Nanocomposite membranes for water separation and purification: Fabrication, modification, and applications. Separation and Purification Technology, 213(September 2018), 465-499. https://doi.org/10.1016/j.seppur.2018.12.050

Fathollah, P., Mortazavi. Y., Jafari. S. H., Khodadadi. A. (2015) Combination of plasma functionalization and phase inversion process techniques for efficient dispersion of MWCNTs in polyamide 6: assessment through morphological, electrical, rheological and thermal properties. Polymer-Plastics Technology and Engineering, v. 54, p. 632-638. https://doi.org/10.1080/03602559.2014.974269

Field, R. W. \& Lipnizki, F. Membrane Separation Processes An Overview. In: Field, R. W., Bekassy-Molnar, E., Lipnizki, F., \& Vatai, G. (2017). Engineering Aspects of Membrane Separation and Application in Food Processing, Boca Raton: CRC Press, ISBN 9781420083637

Fonseca Couto, C., Lange, L. C., \& Santos Amaral, M. C. (2018). A critical review on membrane separation processes applied to remove pharmaceutically active compounds from water and wastewater. Journal of Water Process Engineering (Vol. 26, p. 156-175). Elsevier Ltd. https://doi.org/10.1016/j.jwpe.2018.10.010

Gebreslase, G. A. (2018). Review on Membranes for the Filtration of Aqueous Based Solution: Oil in Water Emulsion. Journal of Membrane Science \& Technology, 08(02). https://doi.org/10.4172/2155-9589.1000188

Ghanbari Shohany, B., \& Khorsand Zak, A. (2020). Doped ZnO nanostructures with selected elements - Structural, morphology and optical properties: A review. In Ceramics International (Vol. 46, Número 5, p. 5507-5520). Elsevier Ltd. https://doi.org/10.1016/j.ceramint.2019.11.051 
Goh, P. S., \& Ismail, A. F. (2018). A review on inorganic membranes for desalination and wastewater treatment. In Desalination (Vol. 434, p. 60-80). Elsevier B.V. https://doi.org/10.1016/j.desal.2017.07.023

Gohil, J. M., \& Ray, P. (2017). A review on semi-aromatic polyamide TFC membranes prepared by interfacial polymerization: Potential for water treatment and desalination. In Separation and Purification Technology (Vol. 181, p. 159-182). Elsevier B.V. https://doi.org/10.1016/j.seppur.2017.03.020

Habert, A. C.; Borges C. P. \& Nobrega, R. (2006) Processos de Separação por Membranas. Rio de Janeiro: E-papers. ISBN: 85-7650-085-X

Hairom, N. H. H., Mohammad, A. W., \& Kadhum, A. A. H. (2014). Effect of various zinc oxide nanoparticles in membrane photocatalytic reactor for Congo red dye treatment. Separation and Purification Technology, 137, 74-81. https://doi.org/10.1016/j.seppur.2014.09.027

He, X., Chen, C., Jiang, Z., \& Su, Y. (2011). Computer simulation of formation of polymeric ultrafiltration membrane via immersion precipitation. Journal of Membrane Science, 371(1-2), 108-116. https://doi.org/10.1016/j.memsci.2011.01.016

Hendricks. D. (2011). Fundamentals of Water Treatment Unit Processes: Physical, Chemical, and Biological. Boca Raton: IWA Publishing. ISBN: 13: 978-1$4200-6192-5$

Isawi, H. (2018). Development of thin-film composite membranes via radical grafting with methacrylic acid/ $\mathrm{ZnO}$ doped TiO2 nanocomposites. Reactive and Functional Polymers, 131(September), 400-413. https://doi.org/10.1016/j.reactfunctpolym.2018.08.018

Iulianelli, A., \& Drioli, E. (2020). Membrane engineering: Latest advancements in gas separation and pre-treatment processes, petrochemical industry and refinery, and future perspectives in emerging applications. In Fuel Processing Technology (Vol. 206, p. 106464). Elsevier B.V. https://doi.org/10.1016/j.fuproc.2020.106464

Kahouli, M., Barhoumi, A., Bouzid, A., Al-Hajry, A.,Guermazi, S. (2015). Structural and optical properties of ZnO nanoparticles prepared by direct precipitation method. In Superlattices and Microstructures (Vol. 85, p. 7-23). Academic Press. https://doi.org/10.1016/j.spmi.2015.05.007

Kausar. A .(2017) Phase inversion technique-based polyamide films and their applications: a comprehensive review. Polymer-Plastics Technology and Engineering, v. 56, p. 1421-1437. https://doi.org/10.1080/03602559.2016.1276593

Kazemi, F., Jafarzadeh, Y., Masoumi, S., \& Rostamizadeh, M. (2021). Oil-in-water emulsion separation by PVC membranes embedded with GO-ZnO nanoparticles. Journal of Environmental Chemical Engineering, 9(1), 104992. https://doi.org/10.1016/j.jece.2020.104992

Khulbe, C. K., Feng, C.Y., Matsuura, T.( 2008) Synthetic polymeric membranes: characterization by atomic force microscopy. Berlin: Springer. ISBN: 978-3540-73994-4

Leo, C. P., Cathie Lee, W. P., Ahmad, A. L., \& Mohammad, A. W. (2012). Polysulfone membranes blended with ZnO nanoparticles for reducing fouling by oleic acid. Separation and Purification Technology, 89, 51-56. https://doi.org/10.1016/j.seppur.2012.01.002

Marana, N. L.; Sambrano, J. R.; Souza, A. R. (2010). Propriedades eletrônicas, estruturais e constantes elásticas do ZnO. Química Nova, v. 33, p. 810-815. https://doi.org/10.1590/S0100-40422010000400009

Matsuyama, H., Takida, Y., Maki, T., \& Teramoto, M. (2002). Preparation of porous membrane by combined use of thermally induced phase separation and immersion precipitation. Polymer, 43(19), 5243-5248. https://doi.org/10.1016/S0032-3861(02)00409-3

Mayrinck, C.; Raphael, E.; Ferrari, J. L.; Schiavon, M. A. (2014). Síntese, propriedades e aplicações de óxido de zinco nanoestruturado. Revista Virtual de Química.v. 6, p. 1185-1204. DOI:10.5935/1984-6835.20140078

Mirzaei, H., \& Darroudi, M. (2017). Zinc oxide nanoparticles: Biological synthesis and biomedical applications. In Ceramics International (Vol. 43, Número 1, p. 907-914). Elsevier Ltd. https://doi.org/10.1016/j.ceramint.2016.10.051

Mishra, Y. K., \& Adelung, R. (2018). ZnO tetrapod materials for functional applications. In Materials Today (Vol. 21, Número 6, p. 631-651). Elsevier B.V. https://doi.org/10.1016/j.mattod.2017.11.003

Mulder, M. (1996) Basic Principles of Membrane Technology. Springer Netherlands. Second Edition. Kluwer Academic Publishers. IBSN:079234247X

Nasrollahi, N., Vatanpour, V., Aber, S., \& Mahmoodi, N. M. (2018). Preparation and characterization of a novel polyethersulfone (PES) ultrafiltration membrane modified with a $\mathrm{CuO} / \mathrm{ZnO}$ nanocomposite to improve permeability and antifouling properties. Separation and Purification Technology, 192(June 2017), 369-382. https://doi.org/10.1016/j.seppur.2017.10.034

Nath, K. (2017). Membrane Separation Processes. Second Edition, Delhi: PHI Learning. ISBN: 978-81-2035291-9

Naz, M. Y., Ahmad, S., Shukrullah, S., Altaf, N. U. H., \& Ghaffar, A. (2020). Effect of microwave plasma treatment on membrane structure of polysulfone fabricated using phase inversion method. Materials Today: Proceedings. https://doi.org/10.1016/j.matpr.2020.04.522

Obreja, P., Cristea, D., Dinescu, A., \& Romanițan, C. (2019). Influence of surface substrates on the properties of ZnO nanowires synthesized by hydrothermal method. In Applied Surface Science (Vol. 463, p. 1117-1123). Elsevier B.V. https://doi.org/10.1016/j.apsusc.2018.08.191

Ozgur, U., Alivov, Y. I., Liu, C., Teke, A., Reshchicov, M. A., Dogan, S., Avrutin, V., Cho, S. J., Morkoç, H. (2005). A Comprehensive review of ZnO materials and devices. Journal of Applied Physics, v. 98, p. 1-104. https://doi.org/10.1063/1.1992666

Pabby, A. K., Rizvi, S. S. H. \& Sastre, A. M. (2015). Handbook of Membrane Separations, Boca Raton: CRC Press.

Pal, N., Agarwal, M., Maheshwari, K., \& Solanki, Y. S. (2020). A review on types, fabrication and support material of hydrogen separation membrane. Materials Today: Proceedings, 28, 1386-1391. https://doi.org/10.1016/j.matpr.2020.04.806 
Pan, Z., Song, C., Li, L., Wang, H., Pan, Y., Wang, C., Li, J., Wang, T., \& Feng, X. (2019). Membrane technology coupled with electrochemical advanced oxidation processes for organic wastewater treatment: Recent advances and future prospects. Chemical Engineering Journal, 376, 120909. https://doi.org/10.1016/j.cej.2019.01.188

Park, H. G., \& Khang, D. Y. (2016). Asymmetric porous membranes from binary polymer solution by physical gelation induced phase separation. Polymer, 87, 323-329. https://doi.org/10.1016/j.polymer.2016.02.016

Ponnamma, D., Cabibihan, J. J., Rajan, M., Pethaiah, S. S., Deshmukh, K., Gogoi, J. P., Pasha, S. K. K., Ahamed, M. B., Krishnegowda, J., Chandrashekar, B. N., Polu, A. R., \& Cheng, C. (2019). Synthesis, optimization and applications of ZnO/polymer nanocomposites. Materials Science and Engineering C, 98(December 2018), 1210-1240. https://doi.org/10.1016/j.msec.2019.01.081

Rajakumaran, R., Boddu, V., Kumar, M., Shalaby, M. S., Abdallah, H., \& Chetty, R. (2019a). Effect of ZnO morphology on GO-ZnO modified polyamide reverse osmosis membranes for desalination. Desalination, 467(June), 245-256. https://doi.org/10.1016/j.desal.2019.06.018

Rajakumaran, R., Boddu, V., Kumar, M., Shalaby, M. S., Abdallah, H., \& Chetty, R. (2019b). Effect of ZnO morphology on GO-ZnO modified polyamide reverse osmosis membranes for desalination. Desalination, 467, 245-256. https://doi.org/10.1016/j.desal.2019.06.018

Rajakumaran, R., Kumar, M., \& Chetty, R. (2020). Morphological effect of ZnO nanostructures on desalination performance and antibacterial activity of thinfilm nanocomposite (TFN) membrane. Desalination, 495(April), 114673. https://doi.org/10.1016/j.desal.2020.114673

Shaban, M., AbdAllah, H., Said, L., Hamdy, H. S., \& Abdel Khalek, A. (2015). Titanium dioxide nanotubes embedded mixed matrix PES membranes characterization and membrane performance. Chemical Engineering Research and Design, 95, 307-316. https://doi.org/10.1016/j.cherd.2014.11.008

Sridhar, S., Moulik, S. Tackling Challenging Industrial Separation Problems through Membrane Technology. In: Sridhar, S., Moulik, S. Membrane Processes Pervaporation, Vapor Permeation and Membrane Distillation for Industrial Scale Separations. Hoboken: John Wiley \& Sons, 2019. ISBN: 978-1-119-418351

Takht Ravanchi, M., Kaghazchi, T., \& Kargari, A. (2009). Application of membrane separation processes in petrochemical industry: a review. Desalination, 235(1-3), 199-244. https://doi.org/10.1016/j.desal.2007.10.042

Tawalbeh, M., Al Mojjly, A., Al-Othman, A., \& Hilal, N. (2018). Membrane separation as a pre-treatment process for oily saline water. In Desalination (Vol. 447, p. 182-202). Elsevier B.V. https://doi.org/10.1016/j.desal.2018.07.029

Torres-Trueba, A., Ruiz-Treviño, F. A., Luna-Bárcenas, G., \& Ortiz-Estrada, C. H. (2008). Formation of integrally skinned asymmetric polysulfone gas separation membranes by supercritical CO2. Journal of Membrane Science, 320(1-2), 431-435. https://doi.org/10.1016/j.memsci.2008.04.024

Uragami, T. Science and Technology of Separation Membranes. Chichester: John Wiley \& Sons Ltd, 2017.

Wu, Y., Gao, M., Chen, W., Lü, Z., Yu, S., Liu, M., \& Gao, C. (2020). Efficient removal of anionic dye by constructing thin-film composite membrane with high perm-selectivity and improved anti-dye-deposition property. Desalination, 476, 114228. https://doi.org/10.1016/j.desal.2019.114228

Yong, W. F., \& Zhang, H. (2021). Recent advances in polymer blend membranes for gas separation and pervaporation. In Progress in Materials Science (Vol. 116, p. 100713). Elsevier Ltd. https://doi.org/10.1016/j.pmatsci.2020.100713

Zang, Z., \& Tang, X. (2015). Enhanced fluorescence imaging performance of hydrophobic colloidal ZnO nanoparticles by a facile method. Journal of Alloys and Compounds, 619, 98-101. https://doi.org/10.1016/j.jallcom.2014.09.072

Zhang, X., Wang, Y., Liu, Y., Xu, J., Han, Y., \& Xu, X. (2014). Preparation, performances of PVDF/ZnO hybrid membranes and their applications in the removal of copper ions. Applied Surface Science, 316(1), 333-340. https://doi.org/10.1016/j.apsusc.2014.08.004

Zhu, L., Song, H., Wang, G., Zeng, Z., \& Xue, Q. (2018). Symmetrical polysulfone/poly(acrylic acid) porous membranes with uniform wormlike morphology and $\mathrm{pH}$ responsibility: Preparation, characterization and application in water purification. Journal of Membrane Science, 549, 515-522. https://doi.org/10.1016/j.memsci.2017.12.052 